\title{
Undelayed 3D RO-SLAM based on Gaussian-Mixture and Reduced Spherical Parametrization
}

\author{
Felipe R.Fabresse ${ }^{1}$, Fernando Caballero ${ }^{1}$, Iván Maza ${ }^{1}$ and Aníbal Ollero ${ }^{1}$
}

\begin{abstract}
This paper presents an undelayed range-only simultaneous localization and mapping (RO-SLAM) based on the Extended Kalman filter. The approach is optimized for working in 3D scenarios, reducing the required computational payload at two levels: first, using a reduced spherical state vector parametrization and, second, proposing a new EKF update scheme. The paper proposes a state vector parametrization based on Gaussian-Mixture to cope with the multi-modal nature of range-only measurements and a reduced spherical parametrization of the range sensor positions that allows to shorten the length of the state vector for a given number of hypotheses. The approach is firstly tested and discussed in simulation, followed by experimental results involving a real robot and radio-based range sensors.
\end{abstract}

\section{INTRODUCTION}

Range-only simultaneous localization and mapping (ROSLAM) is an emerging application that aims to localize a mobile system at the same time it maps the position of a set of range sensors. In contrast with others SLAM approaches based on cameras or LIDAR, RO-SLAM has the advantage of integrating non-line-of-sight (NLOS) measurements into the localization and mapping approach when radio-based range sensing is used. In addition, the data association problem is intrinsically solved by using unique identifiers for each range sensor into the system. RO-SLAM poses serious challenges mainly related with its low-informative measurements (distance between two sensing elements) that leads to multiple localization hypotheses that do not fit well with the usual linear/gaussian approximations, the reason why researchers had explored different approximations to solve this problem: neural networks, particle filters, spectral algorithms, etc.

Thus, [1] presents a localization solution where a neural network is used to learn the observation model of a set of fixed radio emitters in order to get the location of a mobile ground robot by learning the signal power associated to each location in a map (fingerprinting methods). The inputs of this neural network are the distance measurements of each sensor and the output is the 2D location of the mobile robot. The mapping problem is not considered in that work. Despite results are very accurate, this method requires a training

This work is partially supported by the ARCAS project (FP7-ICT-20117-287617) funded by the European Commission of the Seventh Framework Programme and two national projects, RANCOM (P11-TIC-7066) and CLEAR (DPI2011-28937-C02-01).

${ }^{1}$ F.R.Fabresse, F.Caballero, I.Maza and A.Ollero are with the University of Seville, Escuela Superior de Ingenieros, Camino de los Descubrimientos s/n, 41092 Sevilla (Spain). felramfabous.es, fcaballero@us.es, imaza@us.es, aollero@cartuja.us.es process for a specific set of nodes, so an online scalable wireless sensor network is not consider in that solution, additionally, this method requires an accurate localization of the robot during such training stage. Authors of [2] proposes a distributed 3D mapping solution using independent particle filters for an initial position estimation on each range sensor. The particle filter takes into account the multi-modality of the observation model since it is a sample-based bayes filter. The position can be estimated with the range measurements between a sensor and a moving robot by registering the position of the robot with a DGPS. An Extended Kalman Filter (EKF) or Unscented Kalman Filter (UKF) is used when the particle filter has converged to a Gaussian distribution. This solution does not take into account the inter-sensor measurements, which can be used to reduce their estimation uncertainty, and also presents a delayed filter initialization, so sensor information cannot be integrated into the filter until it converges to a Gaussian distribution.

Several solutions have been proposed in the last decade for 2D RO-SLAM. In [3] a novel solution is presented using a spectral SLAM algorithm which estimates the mobile robot and nodes location using a SVD decomposition of the observation matrix. This solution presents accurate results similar to those obtained in classical EKF solutions and batch optimization algorithms, and reduces considerably the required computational burden. However, this method is oriented to batch processing, making very difficult its implementation in online RO-SLAM approaches.

A comparison of most common SLAM frameworks is done in [4]. In that paper it is shown how the unscented FastSLAM presents better results over other classical methods based on EKF or UKF. In [5] and [6], a FastSLAM solution is proposed using a particle filter for robot localization and other particle filter for each feature (i.e. for each radio beacon). In [5] an optimization on the particle filter is proposed to estimate the feature position without needing much hypotheses and hence reducing the computational complexity. On the other hand, [6] optimize the problem using an adaptive resampling method for features position estimation. Another FastSLAM solution is considered in [7] and [8], where the authors use a particle filter to initialize an EKF filter for each map feature of the FastSLAM. The use of an EKF for each map feature reduces the computational burden of the problem. The main drawback of those solutions is the delayed initialization of features, so that first measurements are not integrated into the EKF, waiting until an initial trilateration is performed by each individual particle filter. This is an important aspect in RO-SLAM because the 
convergence of map features to Gaussian distributions will significantly depend on the trilateration made by the vehicle.

To avoid this issue, an undelayed method which uses a gaussian-mixture model (GMM) with multiple hypotheses is presented in [9]. The use of a GMM allows to integrate the multi-modality of the observation model for each new map feature, integrating all received measurements since the very first time. As in [10], [9] uses a polar parametrization which adapts better for 2D range-only localization problems. The use of a GMM to model the multi-modality of the observation model is also employed in [11], but in this case a FastSLAM solution is used instead. Hence, map features are initialized using multiple hypotheses, and each map feature is integrated in an independent EKF. Despite the efficiency of this solution, inter-sensor measurements cannot be integrated taking into account the correlation between each pair of detected map features since the FastSLAM framework considers map features completely independent.

This paper proposes extending the $2 \mathrm{D}$ solution presented in [9] to 3D RO-SLAM. On the advantages, this method allows integrating range measurements between sensors (not only between vehicle and sensors), a key issue in RO-SLAM as concluded in [12]. Another important advantage, and the main difference with a direct extension of [9] to $3 \mathrm{D}$, is that this solution proposes a state vector parametrization that allows reducing the required computational load, specially in the correction stage of the EKF.

The document is structured as follows. Section 2 will make an overview of the method proposed to solve the RO-SLAM problem. Section 3 will detail each stage of the EKF-based RO-SLAM algorithm. In section 4 and 5 some simulations and experiment results will be presented, and finally some conclusions and future work will be presented in section 6 .

\section{OVERVIEW OF THE METHOD}

As in [9], a centralized EKF will be used, solving the mapping problem with Gaussian Mixture Models (GMMs). Gaussian mixture models allows to model the multi-modality of the observation model, integrating a non-Gaussian distribution into a Gaussian filter like the Extended Kalman Filter (EKF).

Probability mixture models are probability distributions that are combination of other distributions, they form a semiparametric alternative to non-parametric distributions like particle filters, providing a better flexibility and precision when modelling the underlying statistics of range measurements. In Gaussian mixture models each mode $i$ is a normal distribution $\mathcal{N}\left(\mu_{i}, \sigma_{i}\right)$ weighted by $\omega_{i}$, where $0 \leq \omega_{i} \leq 1$ and $\sum_{i=1}^{k} \omega_{i}=1$. So for a random variable $X$ with $k$ modes, the probability mass function $f_{X}(x)$ will look like:

$$
f_{X}(x)=\sum_{i=1}^{k} \omega_{i} \mathcal{N}\left(\mu_{i}, \sigma_{i}\right)
$$

As it is shown in Fig. 1, this work will use a spherical parametrization to extend the polar parametrization idea used in [10] and [9]. To model the parameters of this

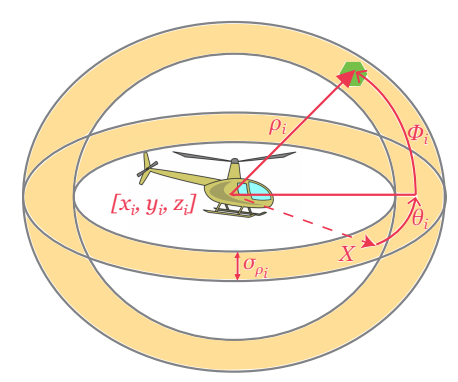

Fig. 1. Spherical parametrization of features. The yellow area represents the uniform spherical distribution where the feature can be located once a first range measurement is received. The green object represents the real position of the range-sensor. The center of the sphere is composed by the position where the robot was located when the first range measurement was received.

spherical parametrization, two Gaussian mixtures are used to represent the azimuth angle $\theta_{i}$ and the elevation angle $\phi_{i}$ of a particular map feature (range-only sensor), so that with a single measurement $r_{i}$ only the distance $\rho_{i}=r_{i}$ and the center of the spehere $\left[x_{i}, y_{i}, z_{i}\right]^{T}$ (i.e. position of the robot at the time $r_{i}$ was received) are known.

Despite a direct extension of the method used in [9] requires a large state vector, which is not efficient when using an Extended Kalman Filter, this work proposes a parametrization reduction which considers $\theta_{i}$ (azimuth) and $\phi_{i}$ (elevation) independent. This assumption will also imply an optimization on the update stage of the EKF since requires smaller matrices to be computed.

As in other approaches like [11], an adaptive initialization will be used in order to improve the initial belief of map features. This adaptive initialization generalize the initialization process used in [9], making the Gaussian Mixture Model fit better to the real uniform distribution of bearing parameters.

On the other hand, a centralized EKF solution will allow to keep the correlation between features estimation, making possible the integration of inter-sensor measurements. This is an important aspect that cannot be provided by FastSLAM solutions where each map feature is assumed to be completely independent from others. The correlation between different map features is interesting specially in those cases where the application has some constraints on the relative position of map features. This is the case when several range sensors are part of the same structural element and, hence, an intrinsic constraint of their relative pose implies a high correlation between them.

\section{EKF-BASED 3D RO-SLAM}

\section{A. State Vector: Reduced spherical parametrization}

The state vector of the EKF is composed by the robot state $\mathbf{x}_{r}$ (for example, $\mathbf{x}_{r}=\left[x_{r}, y_{r}, z_{r}\right]^{T}$ for a 3D Cartesian position of the robot) and the features position $\mathbf{f}_{i}$, so that, for each instant $t$, the state vector will look like:

$$
\mathbf{x}=\left[\mathbf{x}_{r}^{t}, \mathbf{f}_{1}^{t}, \mathbf{f}_{2}^{t}, \ldots, \mathbf{f}_{m}^{t}\right]^{T}
$$

In this state vector, the position of map features is expressed in a reduced spherical representation with respect the 
position $\mathbf{x}_{i}=\left[x_{i}, y_{i}, z_{i}\right]^{T}$ from which the robot received the first range measurement. Thus, when the parameters of the map feature position $i$ are completely known, it is expressed as $\mathbf{f}_{i}=\left[\mathbf{x}_{i}^{t}, \rho_{i}, \theta_{i}, \phi_{i}\right]^{T}$, where $\rho_{i}$ is the distance (first range measurement $r_{i}$ received) between the map feature (range sensor) and the robot, $\theta_{i}$ is the azimuth angle and $\phi_{i}$ is the elevation angle. However, $\theta_{i}$ and $\phi_{i}$ are completely unknown with a single range measurement. Thus, this work propose sampling the space $\theta_{i}$ in $n_{\theta}$ possible samples and $\phi_{i}$ in $n_{\phi}$ possible samples. Although a direct extension of the method proposed in [9] would introduce all $n_{\theta} n_{\phi}$ hypotheses of a feature in the state vector using $4+n_{\theta} n_{\phi}$ parameters, here it is proposed a reduced parametrization that only requires $4+n_{\theta}+n_{\phi}$ parameters to represent the same number of hypotheses. Then, in this work, the state vector of a map feature is expressed with the following reduced spherical parametrization:

$$
\mathbf{f}_{i}=\left[\mathbf{x}_{i}^{t}, \rho_{i}, \theta_{i 1}, \theta_{i 2}, \ldots, \theta_{i n_{\theta}}, \phi_{i 1}, \phi_{i 2}, \ldots, \phi_{i n_{\phi}}\right]^{T}
$$

While the representation of the state vector using a direct extension of [9] would be expressed as:

$$
\mathbf{f}_{i}=\left[\mathbf{x}_{i}^{t}, \rho_{i}, \theta_{i 1}, \phi_{i 1}, \theta_{i 2}, \phi_{i 2}, \ldots, \theta_{i k}, \phi_{i k}\right]^{T}
$$

Here, samples $\theta_{i j_{\theta}}$ and $\phi_{i j_{\phi}}$, together with their weights $\omega_{\theta_{i j_{\theta}}}$ and $\omega_{\phi_{i j_{\phi}}}$, compose a pair of Gaussian Mixture Models such as the probability mass function of $\theta_{i}, f_{\theta_{i}}(x)$, will be uniformly distributed in the range of $[0,2 \pi)$ and the probability mass function of $\phi_{i}, f_{\phi_{i}}(x)$, will be uniformly distributed in the range of $(-\pi / 2, \pi / 2)$ :

$$
\begin{aligned}
& f_{\theta_{i}}(x)=\mathcal{U}(0,2 \pi) \approx \sum_{j_{\theta}=1}^{n_{\theta}} \omega_{\theta_{i j_{\theta}}} \mathcal{N}\left(\theta_{i j_{\theta}}, \sigma_{\theta_{i j_{\theta}}}\right) \\
& f_{\phi_{i}}(x)=\mathcal{U}\left(-\frac{\pi}{2}, \frac{\pi}{2}\right) \approx \sum_{j_{\phi}=1}^{n_{\phi}} \omega_{\phi_{i j_{\phi}}} \mathcal{N}\left(\phi_{i j_{\phi}}, \sigma_{\phi_{i j_{\phi}}}\right)
\end{aligned}
$$

With this reduced representation, the combination of all samples as pairs $\left\{\theta_{i j_{\theta}}, \phi_{i j_{\phi}}\right\}$ composes the complete uniform sphere distribution of hypotheses as depicted in Fig. 2 for 15 hypotheses ( $5 \theta_{i j_{\theta}}$ samples and $3 \phi_{i j_{\phi}}$ samples). One of the main issues to properly cover this uniform spherical distribution with the proposed parametrization is the initialization stage, which will be detailed in the following sections together with prediction and update stage and a method to prune hypotheses.

\section{B. Feature initialization}

Once the first measurement $r_{i}$ of a range sensor $i$ has been received, the distribution of all possible azimuth $\left(\theta_{i}\right)$ and elevation $\left(\phi_{i}\right)$ angles are uniformly distributed around a sphere centered in the current robot position $\left[x_{i}, y_{i}, z_{i}\right]^{T}$ as depicted in Fig. 1. This uniform distribution on $\theta_{i}$ and $\phi_{i}$ can be approximated by two independent GMMs using (5) and (6) respectively. Then, each hypotheses of a map feature is composed by the parameters $x_{i}, y_{i}, z_{i}, \rho_{i}$ and a combination $\left\{\theta_{i j_{\theta}}, \phi_{i j_{\phi}}\right\}$ from the set of all combinations of $\theta_{i}$ and $\phi_{i}$ samples.

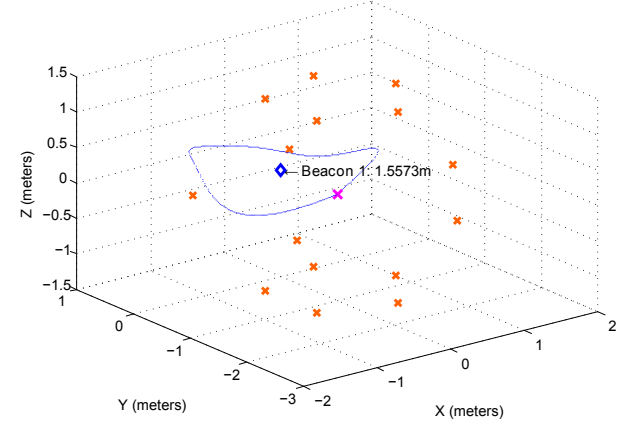

Fig. 2. Filter initialization for 15 hypotheses with a range measure of about 1 meter.

With this parametrization, the number of hypotheses $h$ are related with the number of samples in $\theta_{i}\left(n_{\theta}\right)$ and the number of samples in $\phi_{i}\left(n_{\phi}\right)$ as $h=n_{\theta} n_{\phi}$, where $n_{\theta}=\left\lceil\sqrt{2 h^{*}}\right\rceil$ and $n_{\phi}=\left\lceil n_{\theta} / 2\right\rceil$. The number of optimal hypotheses $h^{*}$ is initialized from the first distance measurement $r_{i}$ received as $h^{*}=4 \pi r_{i}^{2} d$, where $d$ is the optimal density of hypotheses. Different experiments showed that $d=0.18$ is a good value to initialize the number of hypotheses. This value was selected empirically performing several simulations where the robot follows a circular trajectory in plane $X Y$ and moving with a sinusoidal trajectory in $\mathrm{Z}$ axis, the range sensor was placed in the middle of the circular trajectory using a fixed value of $\rho$ and different values of $h$. Then, the optimal hypotheses density is selected considering the hypotheses absolute error and the computational load in each simulation. Table I shows some results of these experiments.

TABLE I

EXPERIMENTAL RESULTS FOR OPTIMAL HYPOTHESES DENSITY SELECTION USING ONE RANGE SENSOR WITH $\sigma_{\rho}=50 \mathrm{~cm}$

\begin{tabular}{|c||c||c||c|}
\hline$\rho$ (meters) & Num.Hypotheses & Error $(\mathrm{cm})$ & Hypotheses Density \\
\hline \hline 5 & 62 & 3.8 & 0.19 \\
10 & 240 & 9.3 & 0.18 \\
20 & 1200 & 12.5 & 0.23 \\
40 & 3000 & 11.56 & 0.15 \\
60 & 6500 & 24.2 & 0.15 \\
\hline
\end{tabular}

Once the number of $\theta_{i}$ and $\phi_{i}$ samples is defined, and hence, the number of hypotheses $h$ too, the next step is to define the value of weights $\omega_{\theta_{i j_{\theta}}}$ and $\omega_{\phi_{i j_{\phi}}}$, the mean value of each mode $\theta_{i j_{\theta}}$ and $\phi_{i j_{\phi}}$, and their associated deviation $\sigma_{\theta_{i j_{\theta}}}$ and $\sigma_{\phi_{i j_{\phi}}}$. As the GMM should approximate an uniform distribution, the values of $\omega_{\theta_{i j_{\theta}}}$ and $\omega_{\phi_{i j_{\phi}}}$ are set up as $\omega_{\theta_{i j_{\theta}}}=1 / n_{\theta}$ and $\omega_{\phi_{i j_{\phi}}}=1 / n_{\phi}$.

The mean value of each Gaussian mode should be placed uniformly with $n_{\theta}$ modes on the range $[0,2 \pi)$ for $\theta_{i}$ and $n_{\phi}$ modes on the range $(-\pi / 2, \pi / 2)$ for $\phi_{i}$ as depicted in Fig.2 for 15 hypotheses and a range measurement near to 1 meter (number beside beacon label).

Finally, the standard deviation value of each Gaussian mode $\theta_{i j_{\theta}}$ and $\phi_{i j_{\phi}}$ is identically initialized according to the following expressions: 


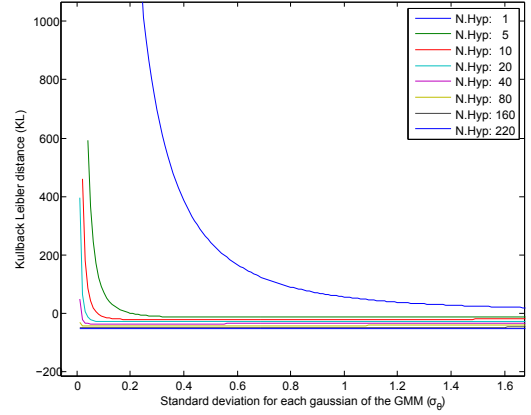

(a) Experiments for $k_{\theta}$

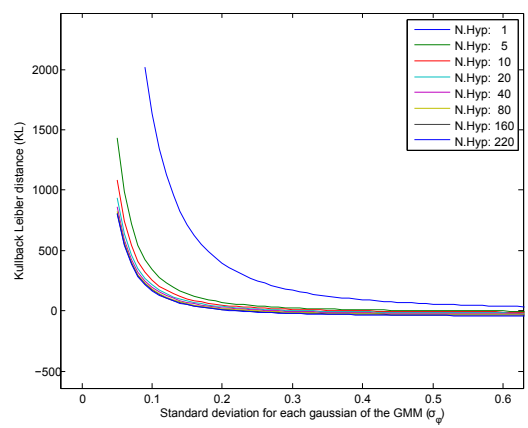

(b) Experiments for $k_{\phi}$

Fig. 3. Experiments performed for the optimal selection of $k_{\theta}$ and $k_{\phi}$ factors. $Y$ axis represents the Kullback-Leibler divergence factor and the $X$ axis represents the standard deviation used in the simulated GMM. Each series represents the simulation for a fixed number of modes in the GMM. (a) shows the results for a GMM in the range $[0,2 \pi)\left(\theta_{i}\right.$ range) and (b) shows the results for a GMM in the range $(-\pi / 2, \pi / 2)\left(\phi_{i}\right.$ range).

$$
\begin{gathered}
\sigma_{\theta_{i j_{\theta}}}=\frac{2 \pi}{k_{\theta} n_{\theta}} \quad j_{\theta}=1, \ldots, n_{\theta} \\
\sigma_{\phi_{i j_{\phi}}}=\frac{\pi}{k_{\phi} n_{\phi}} \quad j_{\phi}=1, \ldots, n_{\phi}
\end{gathered}
$$

Here, values $k_{\theta}$ and $k_{\phi}$ are proportional factors which were selected empirically, performing different simulations where a GMM is compared with the desired uniform distribution. The simulations were performed using different number of modes within the range $[0,2 \pi)$ for $k_{\theta}$ and $(-\pi / 2, \pi / 2)$ for $k_{\phi}$, and different values of the standard deviation $\sigma$ associated to these modes. The GMM obtained in each simulation was compared with the desired uniform distribution using the Kullback-Leibler divergence factor. The results are depicted in Fig. 3, where the $X$ axis represents the standard deviation and the $Y$ axis represents the Kullback-Leibler divergence factor. The values that gave a better fit of the uniform distribution are $k_{\theta}=1.9$ and $k_{\phi}=2.5$.

The main advantages of this initialization process, in contrast to other approaches commented at the introduction, are three: the undelayed initialization which allows the integration of all measurements in the filter since the very first measurement, secondly, it allows the online integration of new range sensors without having to prior select the number of map features that will be used in the EKF, and finally, it adapts the parameters of the GMMs automatically by only employing the first range measurement received from a range sensor.

\section{Update stage}

Once the first measurement of a map feature $\mathbf{f}_{i}$ has been received and its state vector has been initialized, next measurements $r_{i}$ will be used to update map features' belief (EKF filter) and weights $\left(\omega_{\theta_{i j_{\theta}}}\right.$ and $\left.\omega_{\phi_{i j_{\phi}}}\right)$ associated to both GMMs. But, in this work, instead of using $n_{\theta} n_{\phi}$ equations to update the EKF filter for each new range measurement, as it would require a direct extension of method implemented in [9], here only $n_{\theta}+n_{\phi}$ equations are used. Thus, once a new range measurement $r_{i}$ from map feature $\mathbf{f}_{i}$ has been received, the following measurement equation is used to update each $\theta_{i}$ and $\phi_{i}$ sample according to the state vector parametrization described in (2):

$$
r_{i}=\sqrt{\left(x_{f_{i}}-x_{r}\right)^{2}+\left(y_{f_{i}}-y_{r}\right)^{2}+\left(z_{f_{i}}-z_{r}\right)^{2}}
$$

Where $x_{f_{i}}, y_{f_{i}}$ and $z_{f_{i}}$ stand for:

$$
\begin{aligned}
x_{f_{i}} & =x_{i}+\rho_{i} \cos \left(\theta_{i}\right) \cos \left(\phi_{i}\right) \\
y_{f_{i}} & =y_{i}+\rho_{i} \sin \left(\theta_{i}\right) \cos \left(\phi_{i}\right) \\
z_{f_{i}} & =z_{i}+\rho_{i} \sin \left(\phi_{i}\right)
\end{aligned}
$$

Given the previous measurement equation, the problem is how to integrate (9) into the EKF to update each sample independently from each other since (9) depends on both values $\theta_{i}$ and $\phi_{i}$. The solution proposed in this work updates the state vector using $n=n_{\theta}+n_{\phi}$ update equations so that each sample $\theta_{i j_{\theta}}$ is updated using an averaged elevation angle $\overline{\phi_{i}}$ computed according to the current state of the GMM of $\phi_{i}$ as follows:

$$
\overline{\phi_{i}}=\sum_{j_{\phi}=1}^{n_{\phi}} \phi_{i j_{\phi}} \omega_{\phi_{i j_{\phi}}}
$$

Similarly, to update a sample $\phi_{i j_{\phi}}$, the following averaged azimuth angle $\overline{\theta_{i}}$ is used:

$$
\overline{\theta_{i}}=\sum_{j_{\theta}=1}^{n_{\theta}} \theta_{i j_{\theta}} \omega_{\theta_{i j_{\phi}}}
$$

Other problem is how to integrate the associated measurement deviation $\sigma_{r_{i}}$ (see Fig. 1) into these $n$ updates since, as stated in [9], the variance $\sigma_{r_{i}}^{2}$ cannot be used $n$ times from a single measurement $r_{i}$, otherwise, the EKF will tend to diverge. In this work, as in [9], the standard deviation will be split into $n$ variance values $\sigma_{r_{i} j}^{2}$ which are computed as $\sigma_{r_{i} j}^{2}=\sigma_{r_{i}}^{2} / \lambda_{i j}$, where $\lambda_{i j}$ is the proportional likelihood computed for each update equation such us $\sum_{j=1}^{n} \lambda_{i j}=1$. Then, once the $n$ likelihoods $l_{i j}$ have been computed, the proportional likelihood can be computed as follows:

$$
l_{i j}= \begin{cases}p\left(r_{i} \mid \mathbf{x}_{r}^{t}, \mathbf{x}_{i}^{t}, \rho_{i}, \theta_{i j}, \overline{\phi_{i}}\right) & j \in\left[1, n_{\theta}\right] \\ p\left(r_{i} \mid \mathbf{x}_{r}^{t}, \mathbf{x}_{i}^{t}, \rho_{i}, \overline{\theta_{i}}, \phi_{i\left(j-n_{\theta}\right)}\right) & j \in\left[n_{\theta}+1, n_{\theta}+n_{\phi}\right]\end{cases}
$$




$$
\lambda_{i j}=l_{i j} / \sum_{k=1}^{n} l_{i k}
$$

Here, conditional probabilities are modeled with a Gaussian distribution, with mean obtained evaluating eq. (9) for a pair of angles $\left\{\theta_{i j_{\theta}}, \overline{\phi_{i}}\right\}$ or $\left\{\overline{\theta_{i}}, \phi_{i j_{\phi}}\right\}$, and propagating the corresponding state covariances through the Jacobian of the cited equation.

Finally, the last step is to update weights $\omega_{\theta_{i j_{\theta}}}$ and $\omega_{\phi_{i j_{\phi}}}$ associated to both GMM $\theta_{i}$ and $\phi_{i}$. This work uses the likelihood $p\left(r_{i} \mid \mathbf{x}_{r}^{t}, \mathbf{x}_{i}^{t}, \rho_{i}, \theta_{i}, \phi_{i}\right)$ for each $\theta_{i}$ or $\phi_{i}$ sample to update their weights $\omega_{\theta_{i j_{\theta}}}$ and $\omega_{\phi_{i j_{\phi}}}$ respectively. But, now instead of taking the averaged azimuth and elevation angles as in (15), weights are updated as:

$$
\begin{aligned}
& \omega_{\theta_{i j_{\theta}}}=\omega_{\theta_{i j_{\theta}}} \max \left(p\left(r_{i} \mid \mathbf{x}_{r}^{t}, \mathbf{x}_{i}^{t}, \rho_{i}, \theta_{i j_{\theta}}, \phi_{i j_{\phi}}\right) \mid j_{\phi}=1 . . n_{\phi}\right) \\
& \omega_{\phi_{i j_{\phi}}}=\omega_{\phi_{i j_{\phi}}} \max \left(p\left(r_{i} \mid \mathbf{x}_{r}^{t}, \mathbf{x}_{i}^{t}, \rho_{i}, \theta_{i j_{\theta}}, \phi_{i j_{\phi}}\right) \mid j_{\theta}=1 . . n_{\theta}\right)
\end{aligned}
$$

The use of the maximum likelihood makes each weight update for sample $\theta_{i, j_{\theta}}$ or $\phi_{i, j_{\phi}}$ completely independent from other samples. Finally, once all weights are updated using (17) and (18), these weights must be normalized.

\section{Pruning hypotheses}

In order to reduce the computational burden of this method, as map features uncertainty decreases, this paper uses a prune strategy where samples are pruned when satisfying one of the following constraints:

- The associated weight $\omega_{i j}$ is below a certain threshold:

$$
\begin{aligned}
& \omega_{\theta_{i j}} \leq 10^{-11} / h \\
& \omega_{\phi_{i j}} \leq 10^{-11} / h
\end{aligned}
$$

Where $h=n_{\theta} n_{\phi}$ is the current number of hypotheses for a map feature $i$. The threshold was experimentally selected after several simulations of the method.

- The arc length between a sample angle $\theta_{i j_{1}}$ and a set of sample angles $\theta_{i j_{2}}$ or between a sample angle $\phi_{i j_{1}}$ and a set of sample angles $\phi_{i j_{2}}$, with $j_{1} \neq j_{2}$, is lower than the $3 \%$ of $\rho_{i}$ for a feature $i$. The one with higher weight is conserved and those with lower weights are removed.

Notice that, after eliminating a sample $\theta_{i j_{\theta}}, n_{\phi}$ hypotheses are actually being removed ( $n_{\theta}$ hypotheses when removing a sample $\left.\phi_{i j_{\phi}}\right)$.

\section{SIMULATION RESULTS}

In order to test the suitability of the mapping approach, which is the main contribution of this paper, a set of mapping simulations (i.e. the position of the robot is known) have been carried out using only range measurements. The objects simulated are an aerial robot with an embedded radio emitter and a set of free deployed radio beacons.
The simulated wireless sensor network provides the distance measurement from the robot emitter to each beacon using a time-of-flight-based method with a standard deviation of $\sigma_{\rho}=50 \mathrm{~cm}$. Each message received contains the distance measurement and the RFID of the beacon from which the measurement has been received. In order to be as realistic as possible, the maximum transmission rate and the rate of messages sent by each node of the network have also been considered into the simulation.

Figure 4 shows the simulation results for an aerial robot tracking a circular trajectory with a radius of 20 meters. The beacon node is located at the center of this circular trajectory. Thus, the circular trajectory ease the trilateration of beacon on plane XY. But, for a good trilateration of beacon's altitude, the aerial robot moves with a sinusoidal trajectory along $\mathrm{Z}$ axis as it is shown in Fig.4(c).

When the first measurement is received at the beginning of the robot trajectory, the beacon hypotheses are initialized as shown in Fig.4(a) (only the mean of hypotheses are depicted without its associated covariance for simplicity). All modes are initialized with the same weight, this weight is represented according to the color legend shown at right of Fig.4(a). As the robot integrates more measurements into the filter, hypotheses position and their associated weights evolve and some of them are pruned as shown in Fig. 4(b). The weight of each hypotheses has been computed as the combination of associated azimuth and elevation sample weights. At last simulation step, the most likely hypotheses is the only hypotheses depicted in Fig.4(c), whose absolute error evolution is plotted in Fig.4(d). The error has been computed as the euclidean distance between the estimated beacon position and the real one.

\section{EXPERIMENTAL RESULTS}

For real experiments, the complete SLAM problem has been considered so that the mobile robot localization is estimated based on local odometry (using linear and angular speed of the mobile robot). In this experiment, the Pioneer III-AT robot was used with a laptop (8GB of RAM, intel i7 $2.4 \mathrm{GHz}$ quad-core processor). The groundtruth of the robot was registered using a Monte Carlo localization (MCL) algorithm, a laser sensor (Hokuyo UTM-30LX), a map of the CONET testbed and the initial position of the robot in that map. As in simulations, the robot was equipped with a radio emitter which processes distance measurements from 4 different beacons, deployed on an indoor environment of about $15 \mathrm{~m}^{2}$ as shown in Fig.5. The nodes of the network are nanoPAN 5375 RF nodes with the following characteristics:

- ATMega 1284P microcontroller at 20MHz.

- Radio transceiver $2.4 \mathrm{GHz}$ ISM band. Up to $20 \mathrm{~dB}$ transmission power.

- Ranging accuracy of $\mathbf{2} \mathbf{~ m}$ indoors / $1 \mathrm{~m}$ outdoors.

- $128 \mathrm{~KB}$ flash memory for programs and retrieved data.

- Distance measurements computed with the SDS-TWR method, based on the ToF method but without needing any clock synchronization between nodes. 


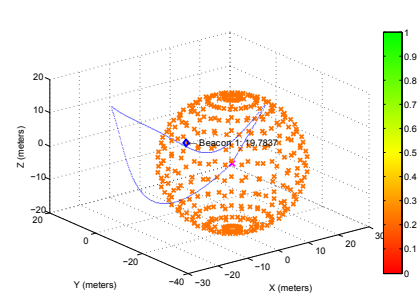

(a)

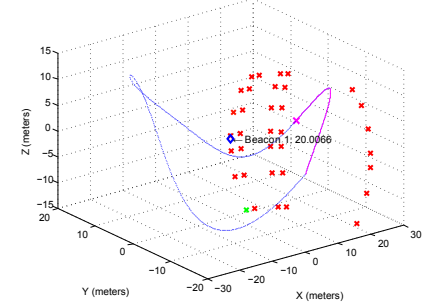

(b)

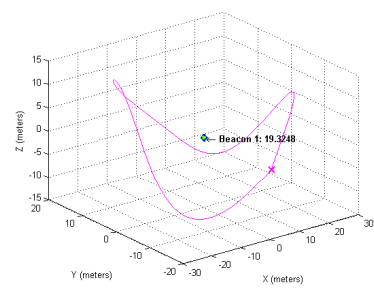

(c)

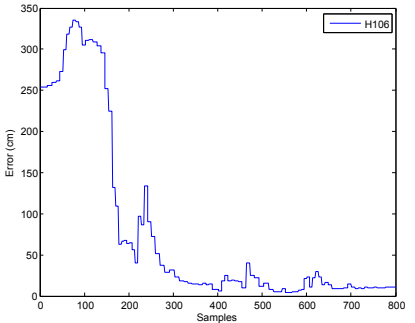

(d)

Fig. 4. Evolution of beacon position hypotheses where the blue rhombus represents the real beacon position, the pink cross represents the robot position and the other crosses are the weighted hypotheses (a) - (c). The complete robot trajectory is depicted as a blue dotted line while the tracked trajectory at current time is depicted as a pink continuous line. The tooltip of the beacon represents the current distance measure received from simulator. (d) represents the evolution of hypotheses absolute error in the last simulation step.

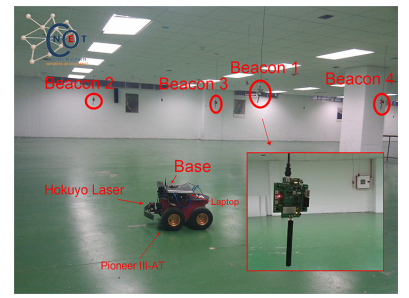

(a) CONET testbed

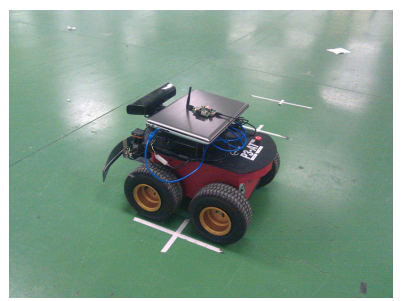

(b) PioneerIII-AT robot equipped with radio emitter on top of the laptop
Fig. 5. Experiments carried out in the CONET testbed at the Escuela de Ingenieros of the University of Seville with a mobile robot equipped with a radio emitter. Beacon emitters are holding from ceil.

As the robot was moving on a plane, only 2D localization was considered for robot localization, while 3D localization was considered for features mapping. Thus, the robot parametrization considered for this experiment was $\mathbf{x}_{r}^{t}=$ $\left[x_{r}, y_{r}, \alpha_{r}\right]^{T}$, where $x_{r}$ and $y_{r}$ are the position of the robot on XY plane, and $\alpha_{r}$ is the robot orientation.

The final SLAM results of this experiment are shown in Fig. 6 and Fig. $7^{1}$. As figures show, virtually all beacons converge to a single solution with an absolute error near to 1 meter. However, despite beacon 3 has not already converged to a single solution due to a bad triangulation over $\mathrm{Z}$ axis and because this node only produced 25 measures over 180 measures produced by other nodes, the error of the most likely hypotheses is lower than 1 meter. As it can be seen, beacons altitude present more variance due to a bad triangulation on $\mathrm{Z}$ axis since the robot is moving with a fixed altitude. Similar error is achieved for robot localization with this SLAM approach, improving the results of deadreckoning method in more that a $50 \%$ as depicted in Fig.6 and Fig. 7.

\section{CONCLUSIONS AND FUTURE WORK}

This paper presented a general solution for the 3D Rangeonly SLAM using a reduced spherical parametrization for the position of map features allowing to reduce the required

\footnotetext{
${ }^{1}$ Complete results are shown in the video attached to this paper which can also be found in the URL: http://grvc.us.es/staff/felramfab/iros2013/video.avi
}

computational load. The solution is based on a centralized EKF-SLAM which includes the position of the robot and the position of all range sensors (map features) which allows the integration of sensor to sensor measurements and not only between robot to sensor keeping the correlation between each pair of sensors and the correlation between sensor to robot. The paper described how the multi-modal belief of the azimuth and elevation angles of a range sensor can be integrated efficiently in a Extended Kalman Filter employing two independent Gaussian Mixtures. The solution proposed not only allows to integrate measurements information since the very first measurement, but also to initialize the position estimation and parametrization of Gaussian Mixtures automatically with only one range measurement. Another important contribution of this paper is the optimization of the EKF correction stage which is based on the consideration of independence between all azimuth and elevation angle parameters.

The paper has presented simulation results to validate the mapping approach showing how this solution is able to feasibly localize nodes of a wireless sensor network (WSN) including the node embedded on the robot. The range sensors considered during the experiments are based on a wireless sensor network where each pair of nodes estimate the distance between them using the SDS-TWR method, which is an improved method of the ToF method. Finally, experimental results for the SLAM approach are shown using the same kind of range sensors considered in simulation but with a real WSN for indoor environments and a mobile robot moving with a fixed altitude. The robot localization results are compared with dead-reckoning method showing the improvement of the method presented. The mapping results also presented good results for 4 beacons and one radio emitter embedded on the robot.

Future research will consider the same problem with not only robot to sensor measurements but also sensor to sensor measurements, which is very interesting when more than one sensor is part of the same structural element (like bars, blocks, ...) as is the case of the ARCAS project. The results will be tested with aerial robots in order to be able to properly estimate the robot and beacons altitude. 


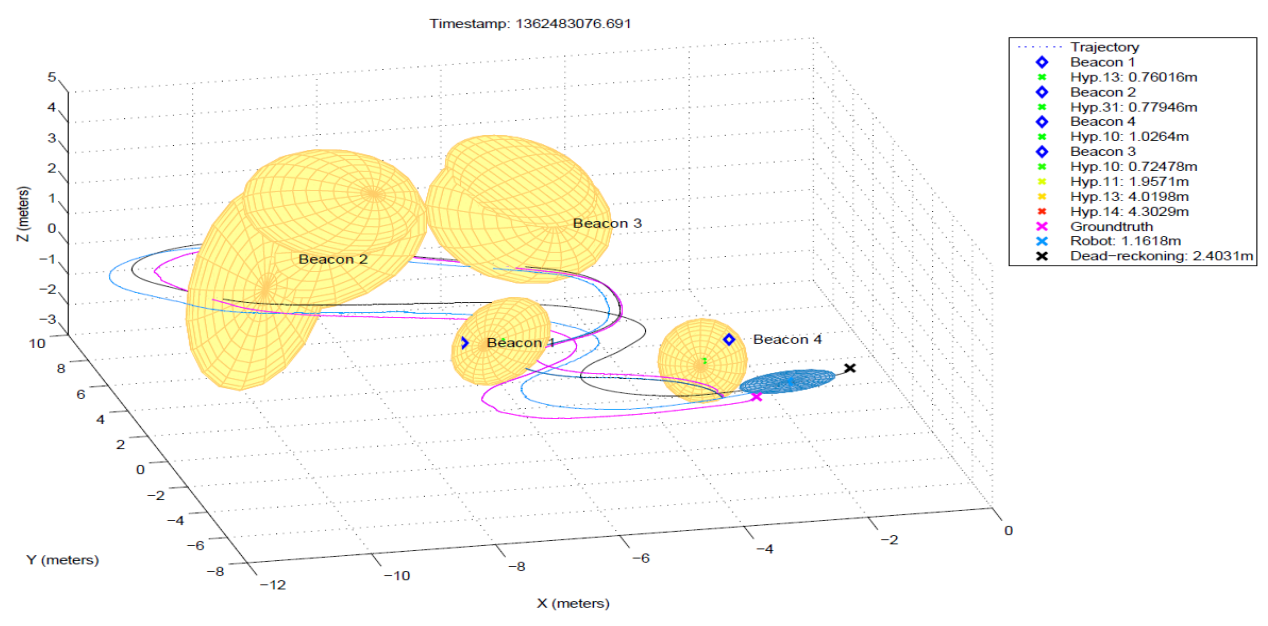

Fig. 6. Final results of proposed 3D SLAM solution with 4 radio-emitters and one mobile robot equipped with a base node. The legend shows the absolute error for each hypotheses and robot localization estimation (SLAM and dead-reckoning). Pink cross and line represent robot groundtruth. Blue cross and line represent robot position estimation using SLAM, where the blue ellipsoid represents the covariance associated. The black cross and line represent the robot position estimation using a dead-reckoning method. Real positions of beacons are depicted with a dark blue rhombus, while beacon hypotheses are depicted with crosses which color, as in Fig.4(a), depends on the hypotheses weight. Covariance associated to each hypotheses is represented with a yellow ellipsoid.

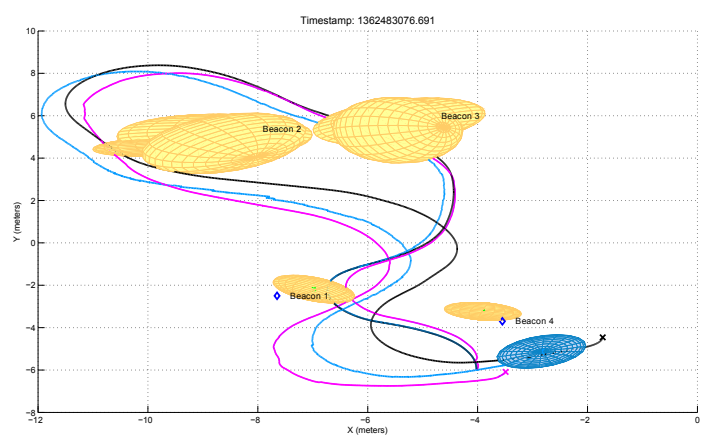

Fig. 7. Final results of proposed 3D SLAM solution (2D representation of Fig.6).

\section{ACKNOWLEDGMENT}

The authors thank the help and support of Arturo Torres González and José Ramiro Martínez de Dios during the experiments performed in this work at the CONET testbed at the School of Engineering (University of Seville).

Fernando Caballero is partially supported by the FROG project (FP7-ICT-2011.2.1) funded by the European Comission.

\section{REFERENCES}

[1] M. Gholami, N. Cai, and R. Brennan, "An artificial neural network approach to the problem of wireless sensors network localization," Robotics and Computer-Integrated Manufacturing, vol. 29, no. 1, pp. 96-109, Feb. 2013. [Online]. Available: http://www.sciencedirect.com/science/article/pii/S0736584512000907

[2] F. Caballero, L. Merino, I. Maza, and A. Ollero, "A particle filtering method for wireless sensor network localization with an aerial robot beacon," in IEEE International Conference on Robotics and Automation, 2008. ICRA 2008, May 2008, pp. $596-601$.

[3] B. Boots and G. J. Gordon, "A spectral learning approach to range-only SLAM," arXiv:1207.2491, July 2012. [Online]. Available: http://arxiv.org/abs/1207.2491
[4] Z. Kurt-Yavuz and S. Yavuz, "A comparison of EKF, UKF, FastSLAM2.0, and UKF-based FastSLAM algorithms," in 2012 IEEE 16th International Conference on Intelligent Engineering Systems (INES), June 2012, pp. $37-43$.

[5] P. Yang, "Efficient particle filter algorithm for ultrasonic sensor-based 2D range-only simultaneous localisation and mapping application," IET Wireless Sensor Systems, vol. 2, no. 4, pp. 394 -401, Dec. 2012.

[6] Z. M. Wang, D. H. Miao, and Z. J. Du, "Simultaneous localization and mapping for mobile robot based on an improved particle filter algorithm," in International Conference on Mechatronics and Automation, 2009. ICMA 2009, Aug. 2009, pp. $1106-1110$.

[7] D. Hai, Y. Li, H. Zhang, and X. Li, "Simultaneous localization and mapping of robot in wireless sensor network," in 2010 IEEE International Conference on Intelligent Computing and Intelligent Systems (ICIS), vol. 3, Oct. 2010, pp. $173-178$.

[8] J.-1. Blanco, J. Gonzlez, and J.-a. Fernndez-madrigal, "A pure probabilistic approach to range-only SLAM," Pasedena Conference Center, California, USA, May 2008.

[9] F. Caballero, L. Merino, and A. Ollero, "A general gaussian-mixture approach for range-only mapping using multiple hypotheses," in Robotics and Automation (ICRA), 2010 IEEE International Conference on, 2010, p. 44044409.

[10] J. Djugash and S. Singh, "A robust method of localization and mapping using only range," in Experimental Robotics, ser. Springer Tracts in Advanced Robotics, O. Khatib, V. Kumar, and G. J. Pappas, Eds. Springer Berlin Heidelberg, Jan. 2009, no. 54, pp. 341-351. [Online]. Available: http://link.springer.com/chapter/10.1007/978-3642-00196-3_40

[11] J.-L. Blanco, J.-A. Fernandez-Madrigal, and J. Gonzalez, "Efficient probabilistic range-only SLAM," in IEEE/RSJ International Conference on Intelligent Robots and Systems, 2008. IROS 2008, Sept. 2008, pp. $1017-1022$.

[12] J. Djugash, "Geolocation with range: Robustness, efficiency and scalability," Ph.D. dissertation, Nov. 2010. [Online]. Available: http://repository.cmu.edu/dissertations/63 